\title{
CALCULATING TIME LAGS FROM UNEVENLY SAMPLED LIGHT CURVES
}

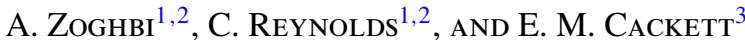 \\ ${ }^{1}$ Department of Astronomy, University of Maryland, College Park, MD 20742-2421, USA; azoghbi@astro.umd.edu \\ 2 Joint Space-Science Institute (JSI), College Park, MD 20742-2421, USA \\ ${ }^{3}$ Department of Physics and Astronomy, Wayne State University, 666 W. Hancock Street, Detroit, MI 48201, USA \\ Received 2013 July 1; accepted 2013 August 27; published 2013 October 10
}

\begin{abstract}
Timing techniques are powerful tools to study dynamical astrophysical phenomena. In the X-ray band, they offer the potential of probing accretion physics down to the event horizon. Recent work has used frequency- and energydependent time lags as tools for studying relativistic reverberation around the black holes in several Seyfert galaxies. This was achieved due to the evenly sampled light curves obtained using XMM-Newton. Continuously sampled data are, however, not always available and standard Fourier techniques are not applicable. Here, building on the work of Miller et al., we discuss and use a maximum likelihood method to obtain frequency-dependent lags that takes into account light curve gaps. Instead of calculating the lag directly, the method estimates the most likely lag values at a particular frequency given two observed light curves. We use Monte Carlo simulations to assess the method's applicability and use it to obtain lag-energy spectra from Suzaku data for two objects, NGC 4151 and MCG-5-23-16, that had previously shown signatures of iron $K$ reverberation. The lags obtained are consistent with those calculated using standard methods using XMM-Newton data.
\end{abstract}

Key words: black hole physics - galaxies: active - galaxies: nuclei - methods: data analysis

Online-only material: color figures

\section{INTRODUCTION}

Variability is ubiquitous in astrophysical phenomena. Observed fluxes are seen to vary on different timescales for different classes of objects. From milliseconds in neutron stars, stellar mass black holes, and gamma ray bursts, timescales progressively increases for different phases of planetary, stellar, and galactic evolution. Variability has been invaluable in understanding the dynamics of many systems that are otherwise observationally inaccessible.

Although a lot of the methodology relies on timedomain analysis, frequency-domain techniques remain the standard tool for characterizing timescale dependencies and (quasi-)periodicity when good data coverage is available. Stellar mass black holes and neutron star low-mass X-ray binaries in particular show a rich phenomenology in the frequency domain that is very tied to state transitions that have distinct spectroscopic signatures (van der Klis 2000; Remillard \& McClintock 2006).

In addition to estimating the power spectral density (PSD) in the frequency domain for a single light curve, other measures exist when multiple, simultaneous (e.g., at different bands or energies) light curves exist. The cross spectrum gives a measure of the combined variability power in two light curves, the coherence measures the fraction of one light curve that can be predicted from the other (Vaughan \& Nowak 1997), and the phase lag gives the relative delay (in units of radians, between $-\pi$ and $\pi$ ) between two light curves as a function of frequency (Miyamoto \& Kitamoto 1989). The phase lag is converted into a time lag (in units of seconds) by dividing the phase lag by the angular frequency of the measurement.

In the X-ray studies of active galactic nucleus (AGN) that motivated this work, frequency-domain techniques have been used extensively to characterize the broadband variability (Papadakis \& McHardy 1995; Uttley et al. 2002; Vaughan et al. 2003; McHardy et al. 2004). Periodicity is generally not seen (see Gierliński et al. 2008 for an exception) and the power spectra are characterized by a power-law of index $\sim 2$ at high frequencies that breaks or bends to $\sim 1$ at a characteristic frequency that appears to scale with mass (McHardy et al. 2006). A standard tool in this case is the fast Fourier transform (FFT), which, for a continuously sampled time series, gives a set of complex numbers at specific frequencies. The periodogram, which estimates the power spectrum and is the squared amplitude of these complex transforms.

Inter-band time delays in the standard X-ray bandpass (0.3-20 keV) in AGNs have just started to be explored in detail. Low frequency hard lags have been seen in early XMMNewton observations (Papadakis et al. 2001; McHardy et al. 2007; Arévalo et al. 2008), where hard bands lag softer bands and the lag magnitude depends on the separation of energies, similar to that in stellar mass black holes (Nowak et al. 1999; Kotov et al. 2001). More recently, high frequency lags have also been seen (e.g., Fabian et al. 2009; Zoghbi et al. 2010; De Marco et al. 2013; Cackett et al. 2013). In this case, the lag can be soft or hard depending on the selected energies, but it is distinguished from the low frequency lags by its energy dependence. The shape of the lag-energy spectra, which gives a measure of the inter-band delays as a function of energy at a particular Fourier frequency, appears to be closely related to the spectroscopic components in a standard spectrum (Zoghbi et al. 2011, 2013; Kara et al. 2013). The fact that the $1-3 \mathrm{keV}$ band leads both the $<1 \mathrm{keV}$ and $>3 \mathrm{keV}$ bands at high frequencies points to a reflection origin, with relativistic reflection being the most plausible explanation. In this case, the reflection spectrum, matched by the lag-energy spectrum, is produced within $\sim 10$ gravitational radii from the event horizon of the black hole and reverberation is produced when the reflecting medium responds to the fast variations of the illuminating source, providing a powerful tool to probe these environments (Reynolds et al. 1999).

Similar to power spectra, time lags in these cases are calculated from the FFT (see Section 2) for continuously sampled 
light curves. The statistical properties of lag measurements in this case are discussed in Nowak et al. (1999). Extending reverberation studies beyond XMM-Newton data is not possible using the standard Fourier techniques because of the inherent non-continuous sampling forced by the low-Earth orbits of other observatories like Suzaku, NuSTAR, and AstroH. The lowest frequencies probed with the standard Fourier techniques are those associated with the orbital period of the satellite, which for the case of low-Earth orbits is higher than the frequency of the interesting reverberation in AGNs. Miller et al. (2010) introduced a method based on likelihood maximization that directly fits for frequency-dependent time lag (along with the power spectrum, based on the work of Bond et al. 1998). In this work, we explicitly discuss this method in detail, assessing its applicability using Monte Carlo simulations. Then, we apply it to Suzaku observations of two objects, NGC 4151 and MCG-5-23-16, that had previously shown relativistic reverberation delays in the iron $K$ band. We start Section 2 by reviewing the standard Fourier techniques for both power spectra and time lags. In Section 3, we describe the formalism of the likelihood method. Section 4 discusses the detailed Monte Carlo simulations of the applicability of the method. The application of the method to Suzaku archival observations of NGC 4151 and MCG-5-23-16 is presented in Section 5.

\section{STANDARD FOURIER TECHNIQUES}

In this section, we briefly review the standard techniques based on the Fourier transform, which are commonly used with evenly sampled light curves. The PSD $\mathcal{P}(f)$ is a property of the stochastic process producing the variability and it gives a measure of the variability power as a function of temporal frequency $f$. It is estimated by calculating the periodogram $I$. If the observed data are in the form of a vector $\boldsymbol{x}$ of length $N$ that gives the count rates at times $t_{i}=i \Delta t$, where $i$ takes the integral values $1,2, \ldots, N$ and $\Delta t$ is the time bin size, the periodogram $I(f)$ is given by the squared amplitude of the discrete Fourier transform of $\boldsymbol{x}$ :

$$
I\left(f_{j}\right)=A\left|\sum_{i=1}^{N} x_{i} e^{i 2 \pi f_{j} t_{i}}\right|^{2}
$$

where $f_{j}=j / N \Delta t$ with $j=1,2, \ldots, N / 2 . A$ is a normalization factor, which we take in this work to be $2 \Delta t / N$ (Vaughan et al. 2003). The periodogram $I$ itself is an inconsistent estimator of $\mathcal{P}$, where its standard deviation at a frequency $f$ is equal to its value (Priestley 1981). The variance is reduced significantly if several frequencies are grouped together (e.g., Papadakis \& Lawrence 1993).

Let us consider a second light curve $y$ that gives the count rate at the same time intervals $t_{i}=i \Delta t$ but in another energy band. The cross spectrum can be estimated as $C(f)=X^{*}(f) Y(f)$, where $X$ and $Y$ are the Fourier transform of $\boldsymbol{x}$ and $\boldsymbol{y}$, respectively, and $X^{*}$ is the complex conjugate of $X$. The cross spectrum is a complex number. Its amplitude is usually expressed in the form of the coherence function $\gamma^{2}(f)=|\langle C\rangle|^{2} /\left(\left\langle|X|^{2}\right\rangle\left\langle|Y|^{2}\right\rangle\right)$ (Vaughan \& Nowak 1997), where the angle brackets denote averaging. The phase of the complex cross spectrum gives the phase lag between the two light curves (Miyamoto \& Kitamoto 1989; Nowak et al. 1999):

$$
\phi(f)=\arg [C(f)] .
$$

The time lag $\tau(f)$ is then obtained by dividing by $2 \pi f$, so that $\tau(f)=\phi(f) / 2 \pi f . \tau(f)$ gives a measure of the time delay between $\boldsymbol{x}$ and $\boldsymbol{y}$ as a function of frequency (or the variability timescale).

The above calculations require the light curve to be evenly sampled so the Fourier transform can be utilized. If this is not the case, other techniques are needed. The following section discusses the method of using the likelihood function to directly fit for the best estimates for the power and cross spectra as well as the phase/time lags directly.

\section{LIKELIHOOD ANALYSIS}

The principal idea behind the method was first presented in the context of X-ray light curves by Miller et al. (2010). Here, we expand it and show explicitly how the method works and perform Monte Carlo simulation to assess its applicability. The method fits for the most likely variability powers and time lags given the observed data. Starting with a model for the power and time lags (which can be of a functional form such as a power law or parameterized with the values of power and time lags in pre-defined frequency bins), a likelihood function that compares the model to the data is constructed (by comparing the auto- and cross-correlations of the data with those expected from the model) and the best estimates are obtained by maximizing this likelihood function. We start in Section 3.1 by applying it to estimating the power spectrum. In Section 3.2, with a simple extension, we use the method to estimate the cross power spectrum and the time lag as a function of Fourier frequency.

\subsection{Power Spectrum Estimate}

As before, the light curve is taken to be $\boldsymbol{x}$ with values $x_{i}$ for $i=1, \ldots, N$, but now $t_{i} \neq i \Delta t$. Following Bond et al. (1998; although that work is for two-dimensional cosmic microwave background data), each $x_{i}$ is the sum of the contribution from the signal $s_{i}$ and noise $n_{i}$. The noise is assumed Gaussian, which is almost always the case given that each $x_{i}$ results from binning measurements obtained at a sampling smaller than $\Delta t$. So, the observed light curve is

$$
\boldsymbol{x}=\boldsymbol{s}+\boldsymbol{n}
$$

with a model correlation matrix given by

$$
\mathrm{C}_{x}=\mathrm{C}_{s}+\mathrm{C}_{n}
$$

where $\mathrm{C}_{s}=\left\langle s_{i} s_{j}\right\rangle$ is the source signal correlation matrix and $\mathrm{C}_{n}=\left\langle n_{i} n_{j}\right\rangle$ is the noise correlation matrix. The angle brackets indicate the ensemble average and we have assumed that the source noise components are independent. Because the measurement errors in light curves are independent, $\mathrm{C}_{n}$ is diagonal with entries $n_{i} n_{i}, i=1, \ldots, N$. In general, if the observations have correlated noise, they can be easily incorporated here by adding non-diagonal elements to $\mathrm{C}_{n}$.

$\mathrm{C}_{s}$ is unknown and its components $c_{i j}$ defined at $\tau=t_{j}-t_{i}$ are related to the underlying power spectrum through the autocorrelation function $\mathcal{A}(\tau)$ :

$$
\langle s(t) s(t+\tau)\rangle=\mathcal{A}(\tau)=\int_{-\infty}^{+\infty} \mathcal{P}(f) \cos (2 \pi f \tau) d f,
$$

using the relation that the autocorrelation is the Fourier transform of the power spectrum and for real functions only the cosine term is included. Now, starting from $\mathcal{P}(f)$ that depends on a number of parameters $\boldsymbol{a}_{\boldsymbol{p}}$ (of length $n_{p}$, say) to be found, 
we construct $\mathrm{C}_{s}$ (following Equation (5)) and calculate the likelihood functions for those parameters:

$$
\mathcal{L}\left(\boldsymbol{a}_{\boldsymbol{p}}\right)=(2 \pi)^{-N / 2}\left|\mathrm{C}_{x}\right|^{-1 / 2} \exp \left[-\frac{1}{2} \boldsymbol{x}^{T} \mathrm{C}_{x}^{-1} \boldsymbol{x}\right],
$$

where the dependence on $\boldsymbol{a}_{\boldsymbol{p}}$ is in $\mathrm{C}_{x}$ through its dependence on $\mathcal{P}$ and $\boldsymbol{x}^{T}$ is the transpose of $\boldsymbol{x}$. Thus, $\mathrm{C}_{x}$ is calculated from the model and $\boldsymbol{x}$ is the data vector. The procedure now is to select a model $\mathcal{P}(f)$ and fit for the parameters $\boldsymbol{a}_{\boldsymbol{p}}$ that maximize the likelihood function in Equation (6). $\mathcal{P}$ can be taken to be a power law or a broken power-law function of $f$. Alternatively, we can fit the band powers directly, taking the powers in pre-defined frequency bands as the parameters $\boldsymbol{a}_{p}$. This is the best option when the intrinsic shape is unknown, which might not be the case for $\mathcal{P}$, but is certainly the case for the frequency-dependent lags $\tau(f)$.

The standard is to maximize $\log (\mathcal{L})$ instead of $\mathcal{L}$. Because of the functional form of the likelihood, the gradient and the second derivatives of the likelihood can be calculated (Bond et al. 1998). An iterative quadratic approximation is then used to find the maximum likelihood. The structure of the loglikelihood function is relatively smooth and converges within a few iterations.

\subsection{Time Lag Estimate}

Extending the previous formalism to include time lags is straightforward. Now, we have another light curve $\boldsymbol{y}=\boldsymbol{r}+\boldsymbol{n}_{y}$ that represents the count rates in a different band, for example. $\boldsymbol{y}$ can be appended to the vector $\boldsymbol{x}$ to give an augmented data vector $\tilde{\boldsymbol{x}}=\left(\begin{array}{l}\boldsymbol{x} \\ \boldsymbol{y}\end{array}\right)$ (Rybicki \& Press 1992). The covariance matrix $\tilde{\mathrm{C}}$ of the new data vector is

$$
\tilde{\mathrm{C}}=\left(\begin{array}{cc}
\mathrm{C}_{x} & \mathrm{C}_{x y} \\
\mathrm{C}_{x y}^{T} & \mathrm{C}_{y}
\end{array}\right)
$$

where $\mathrm{C}_{y}$ is the model covariance matrix of the second light curve $\boldsymbol{y}$ defined in a similar way to Equation (4), with $\mathrm{C}_{r}$ being in general different from $\mathrm{C}_{s}$. The matrix $\mathrm{C}_{x y}$ is the model cross-covariance matrix corresponding to $\left\langle x_{i} y_{j}\right\rangle$. The noise components of $\boldsymbol{x}$ and $\boldsymbol{y}$ are assumed to be independent because the two light curves are produced by independent events in the two bands. The noise components are also independent of the two light curves so that $\left\langle n_{i} x_{j}\right\rangle=0,\left\langle n_{i} y_{j}\right\rangle=0$, etc. Therefore, $\mathrm{C}_{x y}=\left\langle s_{i} r_{j}\right\rangle$.

The cross covariance is related to the cross-power spectrum and the phase lag through the cross-correlation function $\mathcal{X}(\tau)$ :

$$
\langle s(t) r(t+\tau)\rangle=\mathcal{X}(\tau)=\int_{-\infty}^{+\infty} \mathcal{C}(f) \cos (2 \pi f \tau+\phi(\tau)) d f .
$$

The parameters we are interested in are now in the matrix $\tilde{\mathrm{C}}$. The likelihood equation is similar to Equation (6), replacing $\boldsymbol{x}$ and $C$ with $\tilde{\boldsymbol{x}}$ and $\tilde{\mathrm{C}}$, respectively. Again, we use a pre-selected model (e.g., a power law) or choose the powers and lags in pre-defined frequency bins as the parameters of interest. In this work, we choose the latter parameterization. If $n_{B}$ is the number of frequency bins, then we have $n_{p}=4 n_{B}$ parameters: the powers for each light curve, the cross powers, and the phase lags. For this case, the maximization procedure starts with obtaining the PSD values for individual light curves first, then the cross power and phase lags.

In practice, there are also other effects that need to be considered. Aliasing is a consequence of the fact that power cannot be calculated beyond the Nyquist frequency $f_{N}=$ $1 / 2 \Delta t$. The result is that the measured powers at a frequency $f$ also contain contributions from its aliases above $f_{N}$. Fortunately, however, X-ray light curves generally have power-law PSDs, so the power above $f_{N}$ is small. Also, $\Delta t$ is a width of a bin, not the actual sampling time. The binning process is equivalent to convolving the light curve with a binning window $b(t)=1 / \Delta t$ for $-\Delta t / 2<t<\Delta t / 2$ and 0 otherwise. The result is that the $\mathcal{P}$ is multiplied by the Fourier transform of $b(t)$, which is $\sin c^{2}(\pi f \Delta t)$ (e.g., van der Klis 1989). Red noise leak is another problem and it is the result of the finite length of the observation. $\mathcal{P}$ in this case is convolved with the Fourier transform of the window function and mainly causes power below the lowest measured frequency $\left(f_{\min }=1 / T\right.$, where $T$ is the length of the observation) to leak into frequencies above $f_{\min }$. One can explicitly include the convolution of the window in Equations (5) and (8). However, we found that it is computationally easier to include the additional power below $f_{\min }$ in the fit by extending the lowest boundary of the lowest frequency bin to values smaller than $f_{\min }$; this was found to correct for the power biases (see Section 4.1). Extending the first bin to frequencies lower than $f_{\text {min }}$ assumes that the power below $f_{\text {min }}$ does not change significantly, which is a reasonable assumption given that the PSD in almost all cases is a smooth power law.

\subsection{Estimating Uncertainties}

As discussed in Miller et al. (2010), the uncertainties can be estimated as part of the fitting procedure by calculating the Fisher matrix, which is related to the second derivative of the log-likelihood (see the detailed related discussion in Tegmark et al. 1997). The Fisher matrix basically measures how fast on average the likelihood function falls off around its maximum. When the best fit is found, an estimate of the covariance matrix of the parameters is given by the inverse of the Fisher matrix. The variance of the estimates parameters are the diagonal elements of this covariance matrix. These estimates are, however, only a lower limit on the uncertainties when the off-diagonal values are not small (i.e., the parameters are correlated).

The alternative is to step through the parameters, taking the $68 \%$ uncertainty as the value that changes $-2 \log \left(\mathcal{L} / \mathcal{L}_{\text {max }}\right)$ by 1 (Miller et al. 2010). Another approach involves using Monte Carlo Markov Chain (MCMC) in a Bayesian framework to map the full probability space, obtaining probability distributions for the parameters directly. The uncertainties quoted in this work, unless stated otherwise, are the result of stepping through each parameter individually, allowing the rest to change, and taking the error as the value that changes the value of $-2 \log \left(\mathcal{L} / \mathcal{L}_{\max }\right)$ by 1 . This choice works when the number of parameters to be fit is small $\left(n_{p}<\sim 20\right.$, so stepping through parameters is computationally feasible relatively quickly). If the number is large, the best option is to use MCMC to obtain the uncertainties.

\section{SIMULATIONS}

In order to test the above method, we simulate light curves with known underlying power spectra and time delays, introduce gaps, and explore how well they can be recovered. Starting with a functional form for $\mathcal{P}$, we randomize the amplitude and the phase and then inverse Fourier transform to obtain one light curve realization (Timmer \& Koenig 1995). When a second light curve is needed, we shift the phase by the desired amount before performing the inverse Fourier transform. This assumes unity coherence. When fitting real data, the coherence can be estimated from the cross spectrum and the individual power spectra. Poisson noise is added to all light curves. 


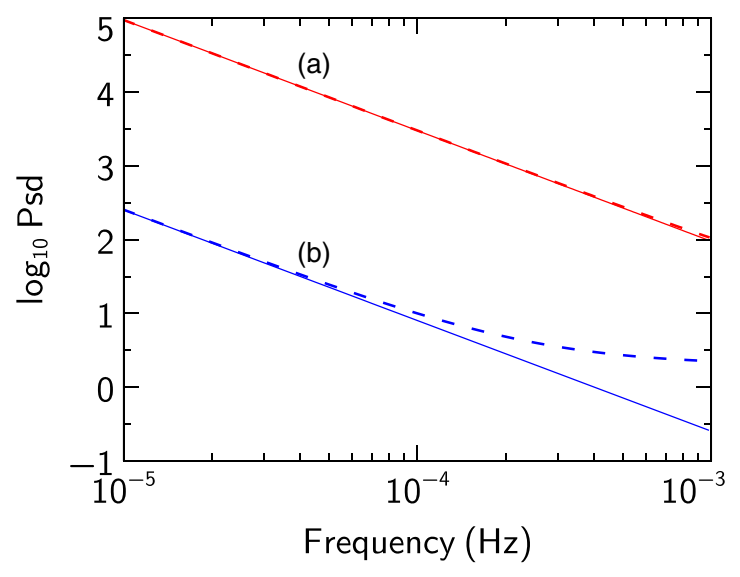

Figure 1. Two cases of base power spectra used in the simulations. Solid lines represent the underlying generating spectrum. Dashed lines include the effect of Poisson noise. Case (a) corresponds to a bright variable (35\% rms) source and case (b) corresponds to a relatively fainter and less variable source $(10 \% \mathrm{rms})$.

(A color version of this figure is available in the online journal.)

In this work, we take the input power spectrum $\mathcal{P}$ to be a broken power law of the form:

$$
\mathcal{P}(f)=A_{b}\left(\frac{f}{f_{b}}\right)^{\alpha}
$$

where $\alpha=-1$ below some break frequency $f_{b}$, and $\alpha=-1.5$ above it. $A_{b}$ is a normalization factor. We take $f_{b}=10^{-6} \mathrm{~Hz}$. This break frequency is consistent with a black hole mass of $\sim 5 \times 10^{7} M_{\odot}$, which is typical of many Seyfert galaxies (McHardy et al. 2006). The lag in the simulated light curves is taken to be constant in phase (at 1 radian), so that the lag scales with $\tau(f) \propto f^{-1}$. For the gaps, we tried different patterns, as will be discussed. The most relevant given X-ray observations are those that have a period of $\sim 1.6 \mathrm{hr}$, typical of low-Earth orbit observations. Throughout the following simulations, we study two cases: $A_{b}=3 \times 10^{6}$ and $A_{b}=8 \times 10^{3}$, representing the high and low power cases, respectively. The count rates for the two cases are 5 and $1 \mathrm{~s}^{-1}$, respectively. These are chosen as typical values for a bright variable and relatively faint, less variable sources, corresponding to rms variabilities of $\sim 35 \%$ and $10 \%$ in each case. For each of these two cases, we run simulations with and without gaps. The simulations without gaps are used for comparison and consistency checks. All the simulated light curves are equivalent to an exposure of $200 \mathrm{ks}$. In simulations with gaps, we discuss both on-source and total exposures of $200 \mathrm{ks}$, as detailed below.

\subsection{Power Spectra}

First, we discuss estimating the power spectrum, starting with a simple, high power, high signal to noise case without including gaps to use as a proof of concept. We simulated more than 2000 light curve realizations from case (a) PSD with a $1 \mathrm{~s}$ sampling rate, added Poisson noise, and then binned the light curves in $512 \mathrm{~s}$ bins. We used frequency bins that give, in the case of even sampling, at least 10 Fourier frequency points per bin. The same experiment is repeated for the low-rms case. The model PSDs and typical light curve realizations for these two cases are shown in Figures 1 and 2.

The result is summarized in Figure 3, where we show the ensemble-averaged measured power in nine frequency bins, along with histogram distributions for two selected frequency bins. Each simulated light curve gives an estimate of the power

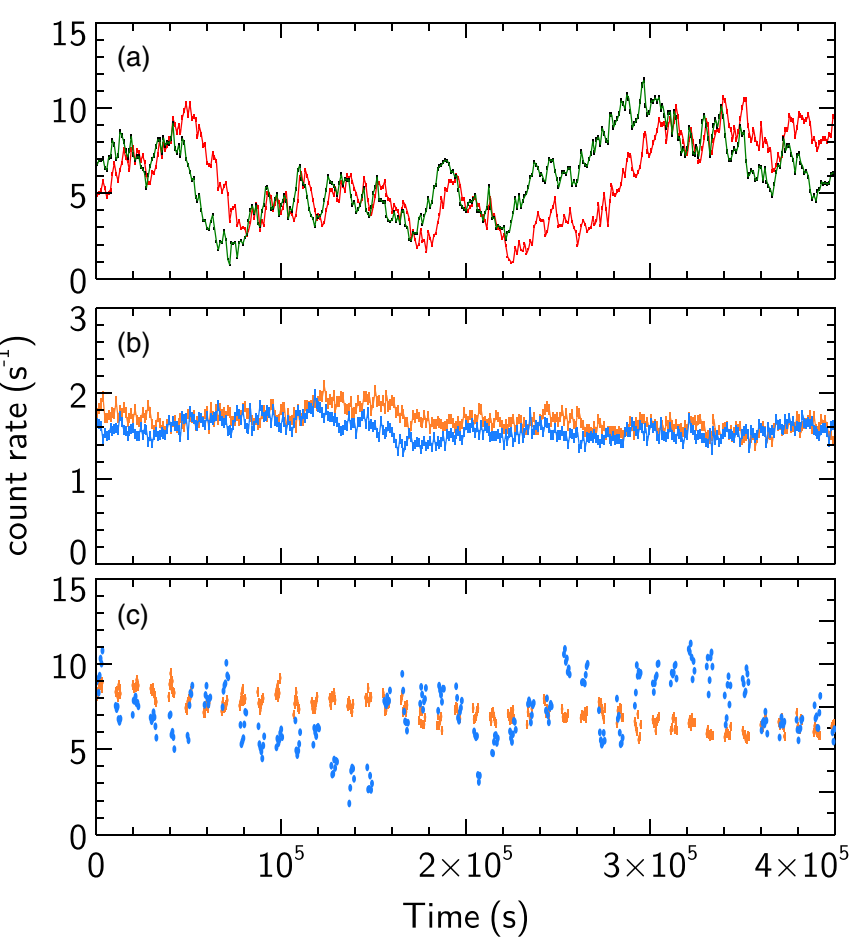

Figure 2. Typical light curve realizations from case (a) (panel (a)) and (b) (panel (b)) in Figure 1. In each case, the second light curve is delayed by 1 radian with respect to first. Panel (c) shows typical light curves with gaps for the two cases. The $y$-axis is similar to panels (a) and (b).

(A color version of this figure is available in the online journal.)

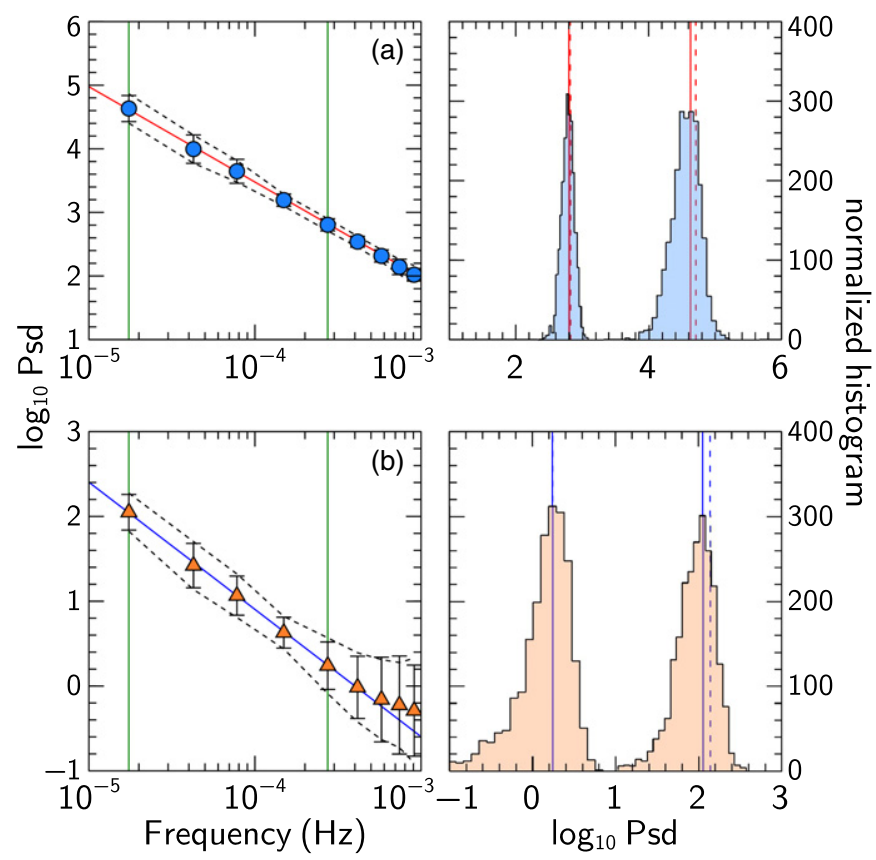

Figure 3. Left: the average estimated PSD for the high- (a) and low-rms (b) cases without including gaps. Right: the histogram of the values for two selected frequency bands (marked with vertical lines in the left panel). For each of the high- and low-rms cases, more than 2000 separate light curves are simulated. For each one, the power is estimated at nine frequency bins. The means of the resulting distributions are plotted in the left panel. Their errors bars represent the standard deviation of the distribution. The average errors from the 2000 estimates are plotted as the dotted lines above and below the best estimate. The solid line is the value of $\mathcal{P}$ (i.e., the input) at that frequency. The frequency error bars representing the width of the bin are omitted for clarity. The vertical lines in the histogram plots represent the mean (the solid lines) and input model (the dotted lines).

(A color version of this figure is available in the online journal.) 
spectrum at the nine frequencies and their uncertainties. The left panel of Figure 3 shows the means of these estimates (points). The errors on those points are taken to be the standard deviation of the estimates around the mean. The averages of the measured uncertainties are also plotted as the dotted lines around the best estimates. The right panel shows the distribution of the 2000 values for two selected frequency bins (first and fifth bins).

There are several points to note from Figure 3. The power spectrum is well-recovered both for the high- and low-rms cases, even in noise-dominated bands in the low-rms case $\left(>2 \times 10^{-4} \mathrm{~Hz}\right.$; see Figure 1$)$. The noise in the light curves is accounted for automatically ( $\boldsymbol{n}$ and $\mathrm{C}_{n}$ in Equations (3) and (4)), so that the measured PSD is the underlying, noiseless $\mathcal{P}$. The estimates are nearly Gaussian, particularly at intermediate frequencies. The distribution tails at the lowest frequencies are consistent with expectations from standard Fourier analysis for bins with a small number of averaged frequencies (e.g., Papadakis \& Lawrence 1993). The shape of the distribution depends essentially on the effective number of independent frequencies present in the light curve. The central limit theorem ensures that when a relatively large number is averaged, the distribution is Gaussian. If the number is small, an estimate is obtained and the errors may not be Gaussian, but the formalism presented here allows us to also estimate the probability distribution of the lags using either direct evaluation of the likelihood function or through MCMCs assuming some priors.

It is also clear that the method gives unbiased, consistent estimates of the power. The plot also shows that the uncertainty estimates (dotted envelopes), discussed in Section 3.3 and taken here as the average of individual uncertainties, are very consistent with the standard deviation of an ensemble of estimates. In fact, for the cases of Figure 3 where no gaps are included, the frequencies are independent and so the uncertainties taken directly from the Fisher matrix and those estimated by stepping through parameter space are the same.

Similar simulations were performed for the low- and highrms cases (as defined in Section 4 and Figure 1) considering light curves with gaps. For comparison, we simulate light curves where the length of observation is $200 \mathrm{ks}$ and also light curves where the on-source exposure is $200 \mathrm{ks}$. The gaps are generated randomly assuming that both the length of the data stream and the gaps are Gaussian random variables with means of 5700 and $4000 \mathrm{~s}$, respectively, with a standard deviation of $100 \mathrm{~s}$. These gap patterns roughly resemble those usually encountered in Suzaku observations and are relevant to NuSTAR and AstroH. Other gap patterns have also been explored and the conclusions are in general the same (with the obvious change of the frequencies affected). The result is plotted in Figure 4.

High- and low-rms cases are plotted in the top and bottom panels, respectively. In each case, the left plot is similar to that in Figure 3. The points and the errors bars are for the case with no gaps, for comparison. The red dotted and green dashed lines are the envelope of the standard deviation of the PSD estimates for light curves with gaps and light curve lengths of $200 \mathrm{ks}$ (hereafter, case G1) and an on-source exposure of $200 \mathrm{ks}$ (hereafter, case G2), respectively.

The gaps have several effects compared with the continuous case. The errors are in general larger because there are less data on the whole, except for the very lowest frequencies where the errors in G2 are smaller than the no gap case because the requirement of a on-source exposure of $200 \mathrm{ks}$ means that
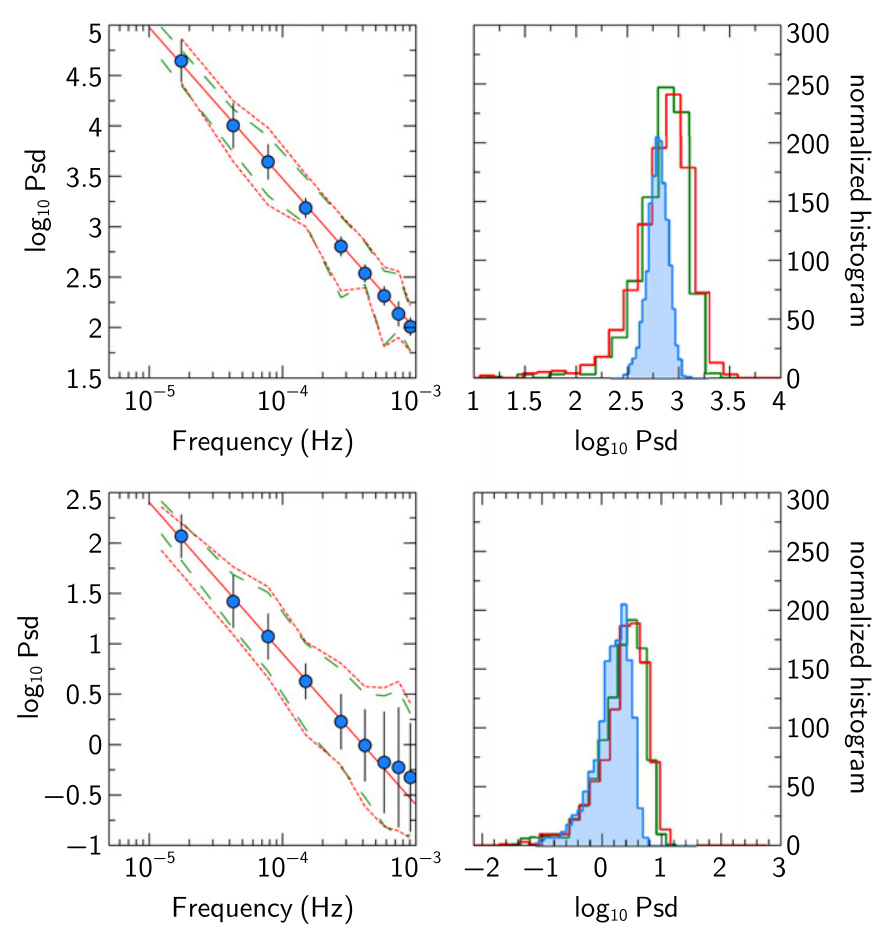

Figure 4. Left: the average estimated power spectra (similar to Figure 3) including light curves with gaps for high (top panel) and low (bottom panel) rms cases. The points represent the distribution means for the case of no gaps (similar to Figure 3) and the error bars are the standard deviation around the mean. The red dotted envelope is the standard deviation of the estimates including gaps with the light curves of length $200 \mathrm{ks}$ (data + gaps, G1). The green dashed envelope is the standard deviation of the estimates for light curves with gaps, but with on-source exposure of $200 \mathrm{ks}$ (data only, G2). Right: a histogram distribution of the estimates at the fifth frequency bin $\left(\sim 2 \times 10^{-4} \mathrm{~Hz}\right)$, which corresponds roughly to the periodicity of the gaps. The blue line is for light curves without gaps. The red line is for the case of a total exposure of $200 \mathrm{ks}$ and the green line is for the case of an on-source exposure of $200 \mathrm{ks}$.

(A color version of this figure is available in the online journal.)

there are more low frequency data. Also, the errors for both G1 and $\mathrm{G} 2$ are larger for frequencies close to the gap periodicity. The reason is that information on those frequencies is missing because of the gaps. This is a general result that we found throughout the simulations and it shows that the periodic gaps cause the uncertainties at the frequency corresponding to the gap periodicity $\left(\sim 1 \times 10^{-4} \mathrm{~Hz}\right)$. G1 has about $60 \%-70 \%$ less exposure and its errors are slightly larger than those of $\mathrm{G} 2$ (the difference for a single frequency band is not huge, but all frequency bins are affected). The distribution histograms for the frequency bin closest to the gap frequencies are also plotted in Figure 4.

\subsection{Time Lag}

An analysis similar to that presented in Section 4.1 was extended to include time lags. Figure 2 show typical light curves pairs for the high- and low-rms cases defined in Section 4, where, for each pair, the second light curve is shifted with a phase of 1 radian. The results are presented in Figure 5. Although the presented simulations are for the case of a constant phase lag of 1 radian, we tested for other forms (e.g., a constant time lag, a time lag that has a functional dependence on $f$, etc.) and the results are not different from those discussed here.

The Figure 5, analogous to Figure 3, shows the averaged lag calculated from an ensemble of 2000 light curve realizations for the high- and low-rms cases without gaps. The lags are wellrecovered for all frequencies for both cases. The distributions of 


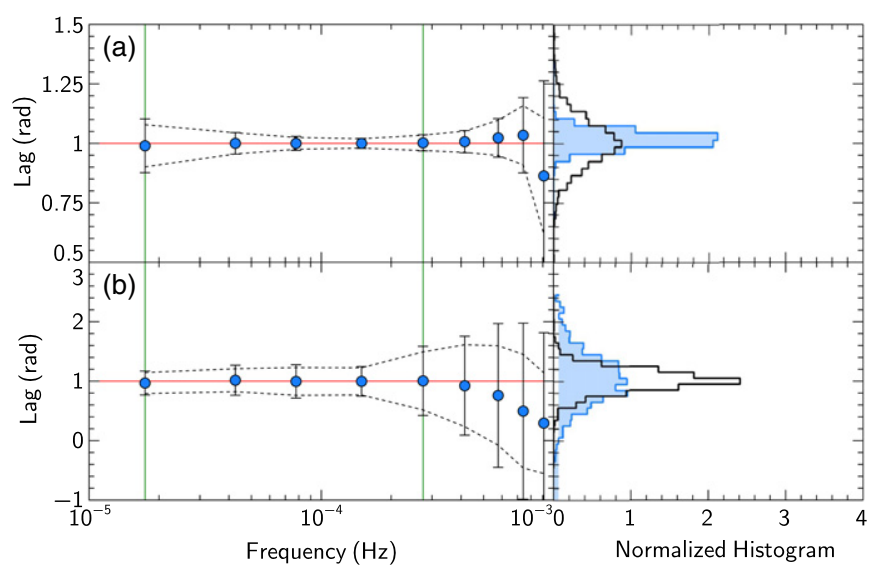

Figure 5. Similar to Figure 3 but now showing lags instead of the PSD. Highand low-rms cases are shown in panels (a, top) and (b, bottom), respectively, for light curves without gaps. The average estimated lag is shown as points. The standard deviations around the mean are shown as error bars. The envelope dotted line shows the average estimated uncertainties. The right panels in each case show the (normalized) number of values histogram for the first (open) and fifth (shaded) frequency bins, marked with vertical lines in the left panels.

(A color version of this figure is available in the online journal.)

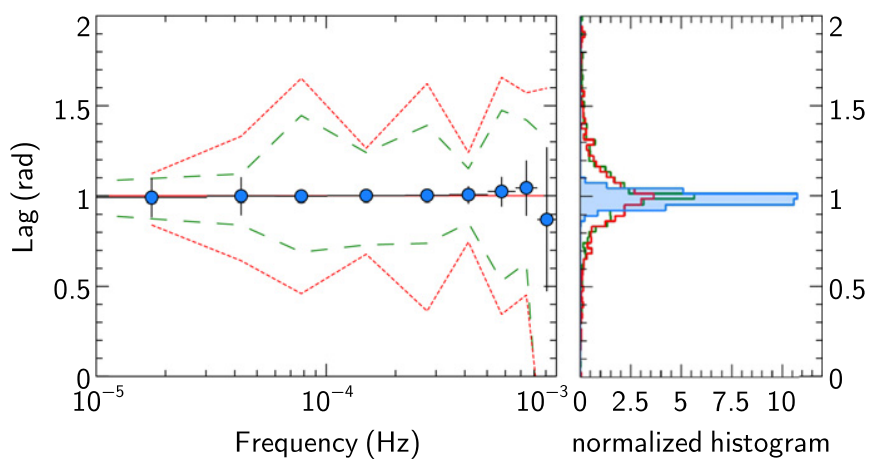

Figure 6. Similar to Figure 5 but now including light curves with gaps for the high-rms case. The average estimated lag for light curves without gaps is shown as points. The standard deviations around the mean are shown as error bars. The envelope dotted line (red) shows the standard deviation for light curves with gaps and lengths of $200 \mathrm{ks}$ (G1). The envelope dashed line (green) is for the case of light curves with gaps and on-source exposures of $200 \mathrm{ks}$ (G2). The right panel shows the corresponding (normalized) number of values histogram for the fifth frequency bin, which corresponds roughly to the frequency of the periodic gaps.

(A color version of this figure is available in the online journal.)

the estimates (shown in Figure 5) are almost perfect Gaussians. The plot also shows that the uncertainty estimates (dotted envelopes), discussed in Section 3.3 and taken here as the ensemble average of individual uncertainties, are also consistent with the standard deviation of an ensemble of estimates. The slight difference at the noise-dominated frequencies (highest frequencies in panel (b) in Figure 5) is an artifact of the simulation, where the noise-dominated parameters sometimes fail to converge; it is therefore hard to obtain uncertainties and those are removed when estimating the average uncertainties. In practical data analysis, one would reduce the number of frequency bins to improve the signal to noise ratio.

Extending the analysis to light curves with gaps is again straightforward (Figure 6). As in the case of power spectra, the errors are larger for light curves with gaps because less information is available in the data. The lowest frequencies are not affected much because the long timescale trends in the light curves are not affected if there are gaps on smaller timescale.

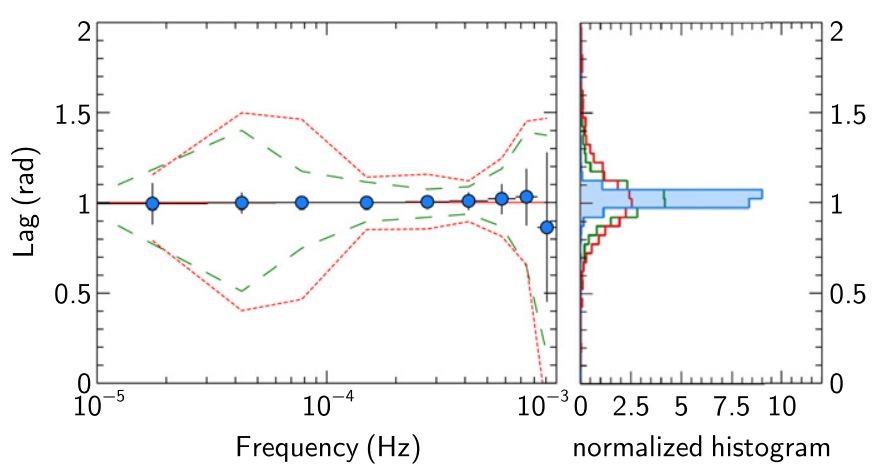

Figure 7. Similar to Figure 6 but with a different gap pattern. The gaps now have a periodicity that corresponds to a frequency of $\sim 7 \times 10^{-5} \mathrm{~Hz}$. The histograms are for the second frequency point.

(A color version of this figure is available in the online journal.)

Table 1

Observations of NGC 4151 and MCG-5-23-16 from the Suzaku Archive Used in This Work

\begin{tabular}{lccc}
\hline \hline Object & Obs. ID & $\begin{array}{c}\text { Exposure } \\
(\mathrm{ks})\end{array}$ & Date \\
\hline NGC 4151 & 701034010 & 125 & $2008 \mathrm{Aug}$ \\
& 906006010 & 60 & $2011 \mathrm{Apr}$ \\
& 906006020 & 60 & $2001 \mathrm{Apr}$ \\
MCG-5-23-16 & 700002010 & 95 & $2005 \mathrm{Dec}$ \\
\hline
\end{tabular}

The periodic gaps have the effect of increasing the uncertainty of the measured lags at frequencies close to the gap frequency and also its harmonics where information is missing. This, combined with the gap randomness (i.e., it is not a single frequency) and frequency binning produces the fluctuations seen in Figure 6. The results for the low-rms case are very similar. The low signalto-noise ratio, however, means that the errors are larger and sometimes simulations are not constrained. Better estimates are obtained when using fewer frequency bins (i.e., improving the signal per bin) and, in this case, the results are similar to those of the high-rms case.

This increased uncertainty at the gap periodicity is further illustrated in Figure 7, which is similar to Figure 6 but for a different gap pattern. Here, the gaps have a periodicity corresponding to a frequency of $\sim 7 \times 10^{-5} \mathrm{~Hz}$. Again, the effect of the gaps is that less information is available to the gap frequency and therefore the uncertainty is larger. The distribution of the estimates is Gaussian or very close to Gaussian in most cases. The power of the likelihood method presented here is that, even in frequency bands where the effective number of independent frequencies is small, one can obtain a direct measure of the probability distribution whether by stepping through the likelihood function or more efficiently by using MCMC.

\section{APPLICATIONS}

In this section, we discuss the application of the above method to calculate time lags in Suzaku observations of two sources: NGC 4151 and MCG-5-23-16. Time delays have been seen in these two objects using XMM-Newton and standard lag calculation methods (Zoghbi et al. 2012, 2013). The observations used in the following discussion are summarized in Table 1.

Data were retrieved from the archives and reduced using HEASOFT 6.13 and the latest calibration files (CALDB version 20130305). Cleaned events files for all X-ray imaging 

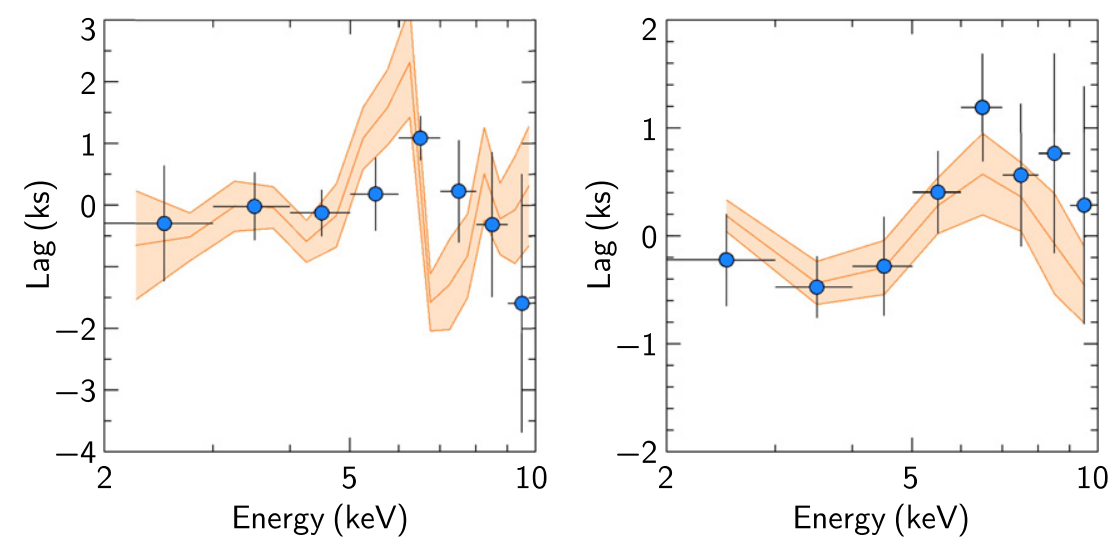

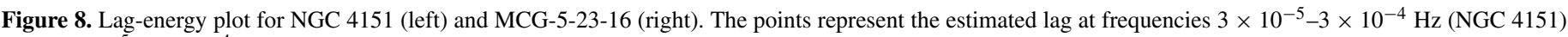

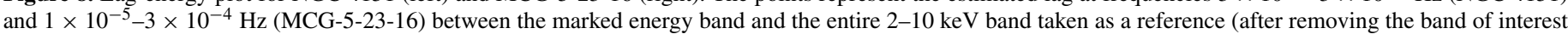

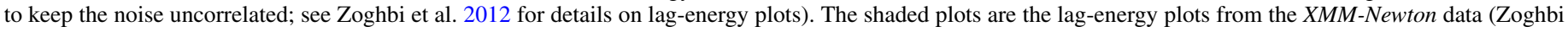
et al. 2012, 2013)

(A color version of this figure is available in the online journal.)

spectrometer (XIS) detectors operational during the observations were produced following the Suzaku user guide. Then, XSELECT was used to extract source and background light curves with time bins of $512 \mathrm{~s}$. The source region in each case was circular with a radius of 3.5 arcmin and background regions were selected from source-free regions on the CCD. In order to study time lags, we extracted light curves in eight energy bins between $2-10 \mathrm{keV}$ in steps of $1 \mathrm{keV}$. Background light curves were than scaled to match the area of the source region before subtracting them from the source light curves. The XIS0 and XIS3 counts were combined to produce a total front-illuminated light curve, while the XIS1 gives a back-illuminated light curve. The two light curves can then be fit simultaneously using the formalism discussed in Section 3.

Figure 8 shows the lag-energy plots for NGC 4151 (left) and MCG-5-23-16 (right), along with plots from previously published XMM-Newton data (Zoghbi et al. 2012, 2013). Each point in the plots is a result of maximizing the likelihood function for the power spectra and phase lags between individual light curves and the total $2-10 \mathrm{keV}$ light curves, excluding the current energies (see Zoghbi et al. 2012 for details on the lag-energy plots). The plotted frequencies are $3 \times 10^{-5}-3 \times 10^{-4} \mathrm{~Hz}$ and $1 \times 10^{-5}-3 \times 10^{-4} \mathrm{~Hz}$, respectively. Although the uncertainties at the highest energies are relatively large, it is clear that there is a structure at 6-7 keV consistent with that seen in the $X M M$-Newton data. The match between the Suzaku and XMMNewton plots in the case of MCG-5-23-16 (Figure 8, right) is remarkable. For the case of NGC 4151, although the shapes are statistically consistent, the lag-energy shape in this source is known to be flux- and frequency-dependent (Zoghbi et al. 2012). The length and quality of the Suzaku observations do not allow for a direct comparison at the same exact frequencies, but the fact that there is a peak at $\sim 6 \mathrm{keV}$ adds further evidence that the iron line is responsible for these lags. A further test is achieved by adding artificial gaps to the XMM-Newton and calculating lags. This, however, reduces the amount of available data and smears the signals out.

The likelihood method allows us to obtain full probability densities for lag estimates and hence quantify directly the significance of any lag detection. For example, Figure 9 shows the probability density of the estimated lag values at $1-2$ and 6-7 keV for the case of MCG-5-23-16, plotted in the right panel of Figure 8. After the best estimates are obtained by maximizing

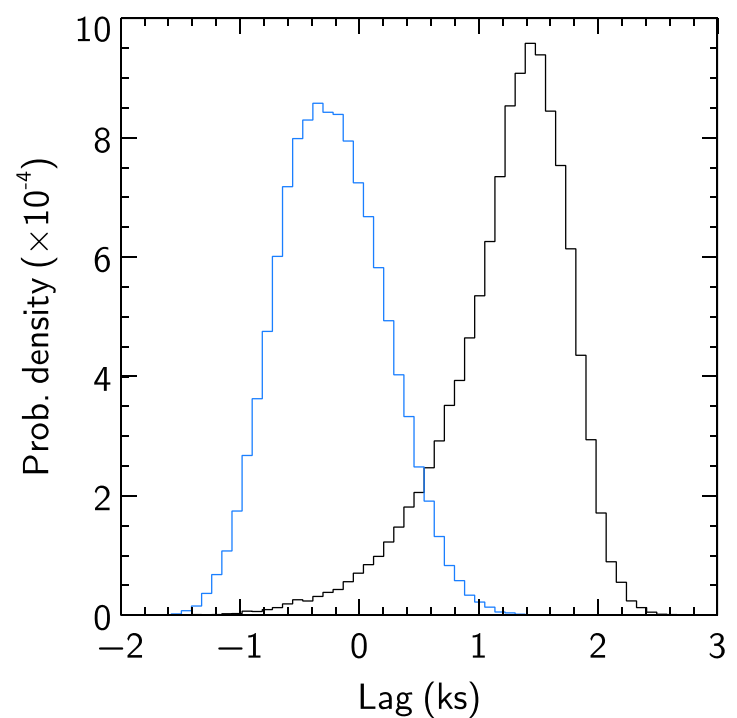

Figure 9. Probability densities for MCG-5-23-16 for the two lag points at 1-2 and 6-7 keV shown in Figure 8 estimated using MCMC.

(A color version of this figure is available in the online journal.)

the likelihood function, the multi-dimensional parameter space is mapped out using MCMC assuming uniform priors for the parameters. The chains were generated with an affine-invariant ensemble sampler (Goodman \& Weare 2010; Foreman-Mackey et al. 2013) and the result is a probability density for each of the estimated values.

\section{SUMMARY}

We presented and discussed a method to calculate frequencydependent power spectra and time lags for unevenly sampled data. The method, first introduced by Miller et al. (2010), relies on likelihood maximization and gives the most likely power and lag estimates given the data. We tested the method using Monte Carlo simulations and showed that the main effect of periodic gaps, typical of low-Earth orbit X-ray observations, is to give unconstrained estimates of the frequency corresponding to the gap periodicity, while information at other frequencies is recovered. We applied the method to Suzaku archival observations of NGC 4151 and MCG-5-23-16 and showed that their lag-energy spectra are consistent with those observed using XMM-Newton, 
giving further support to their interpretations of being due to relativistic reverberation close to black holes.

\section{REFERENCES}

Arévalo, P., McHardy, I. M., \& Summons, D. P. 2008, MNRAS, 388, 211

Bond, J. R., Jaffe, A. H., \& Knox, L. 1998, PhRvD, 57, 2117

Cackett, E. M., Fabian, A. C., Zogbhi, A., et al. 2013, ApJL, 764, L9

De Marco, B., Ponti, G., Cappi, M., et al. 2013, MNRAS, 431, 2441

Fabian, A. C., Zoghbi, A., Ross, R. R., et al. 2009, Natur, 459, 540

Foreman-Mackey, D., Hogg, D. W., Lang, D., \& Goodman, J. 2013, PASP, 125,306

Gierliński, M., Middleton, M., Ward, M., \& Done, C. 2008, Natur, 455, 369

Goodman, J., \& Weare, J. 2010, Commun. Appl. Math. Comput. Sci., 5, 65

Kara, E., Fabian, A. C., Cackett, E. M., Miniutti, G., \& Uttley, P. 2013, MNRAS, 430, 1408

Kotov, O., Churazov, E., \& Gilfanov, M. 2001, MNRAS, 327, 799

McHardy, I. M., Arévalo, P., Uttley, P., et al. 2007, MNRAS, 382, 985

McHardy, I. M., Koerding, E., Knigge, C., Uttley, P., \& Fender, R. P. 2006, Natur, 444, 730

McHardy, I. M., Papadakis, I. E., Uttley, P., Page, M. J., \& Mason, K. O. 2004, MNRAS, 348, 783

Miller, L., Turner, T. J., Reeves, J. N., et al. 2010, MNRAS, 403, 196

Miyamoto, S., \& Kitamoto, S. 1989, Natur, 342, 773
Nowak, M. A., Vaughan, B. A., Wilms, J., Dove, J. B., \& Begelman, M. C. 1999, ApJ, 510, 874

Papadakis, I. E., \& Lawrence, A. 1993, MNRAS, 261, 612

Papadakis, I. E., \& McHardy, I. M. 1995, MNRAS, 273, 923

Papadakis, I. E., Nandra, K., \& Kazanas, D. 2001, ApJL, 554, L133

Priestley, M. B. 1981, Spectral Analysis and Time Series (London: Academic), 890

Remillard, R. A., \& McClintock, J. E. 2006, ARA\&A, 44, 49

Reynolds, C. S., Young, A. J., Begelman, M. C., \& Fabian, A. C. 1999, ApJ, 514,164

Rybicki, G. B., \& Press, W. H. 1992, ApJ, 398, 169

Tegmark, M., Taylor, A. N., \& Heavens, A. F. 1997, ApJ, 480, 22

Timmer, J., \& Koenig, M. 1995, A\&A, 300, 707

Uttley, P., McHardy, I. M., \& Papadakis, I. E. 2002, MNRAS, 332, 231

van der Klis, M. 1989, in Timing Neutron Stars, ed. H. Ögelman \& E. P. J. van den Heuvel (New York: Kluwer), 27

van der Klis, M. 2000, ARA\&A, 38, 717

Vaughan, B. A., \& Nowak, M. A. 1997, ApJL, 474, L43

Vaughan, S., Edelson, R., Warwick, R. S., \& Uttley, P. 2003, MNRAS, 345,1271

Zoghbi, A., Fabian, A. C., Reynolds, C. S., \& Cackett, E. M. 2012, MNRAS, 422, 129

Zoghbi, A., Fabian, A. C., Uttley, P., et al. 2010, MNRAS, 401, 2419

Zoghbi, A., Reynolds, C., Cackett, E. M., et al. 2013, ApJ, 767, 121

Zoghbi, A., Uttley, P., \& Fabian, A. C. 2011, MNRAS, 412, 59 